\title{
Short Wave Infrared Devices Based on HgTe Nanocrystals with Air Stable Performances
}

\author{
Amardeep Jagtap ${ }^{1}$, Nicolas Goubet ${ }^{1,2}$, Clément Livache ${ }^{1,2}$, Audrey Chu ${ }^{1}$, Bertille Martinez ${ }^{1,2}$, \\ Charlie Gréboval ${ }^{1}$, Junling Qu ${ }^{1}$, Erwan Dandeu ${ }^{1}$, Loïc Becerra ${ }^{1}$, Nadine Witkowski ${ }^{1}$, Sandrine \\ Ithurria ${ }^{2}$, Fabrice Mathevet ${ }^{3}$, Mathieu G. Silly ${ }^{4}$, Benoit Dubertret ${ }^{2}$, Emmanuel Lhuillier ${ }^{*}$ \\ 1Sorbonne Université, CNRS, Institut des NanoSciences de Paris, INSP, F-75005 Paris, France \\ ${ }^{2}$ Laboratoire de Physique et d'Etude des Matériaux, ESPCI-Paris, PSL Research University, \\ Sorbonne Université UPMC Univ Paris 06, CNRS, 10 rue Vauquelin 75005 Paris, France. \\ ${ }^{3}$ Sorbonne Université, Institut Parisien de Chimie Moléculaire, UMR 8232, Chimie des \\ Polymères, 4 Place Jussieu, 75252 Paris Cedex 05, France \\ ${ }^{4}$ Synchrotron-SOLEIL, Saint-Aubin, BP48, F91192 Gif sur Yvette Cedex, France.
}

\begin{abstract}
Colloidal quantum dots (CQDs) are candidates of interest for the design of low cost IR detector especially in the short wave infrared (SWIR; 0.8-3 $\mu \mathrm{m}$ ), where the vicinity of the visible range makes the high cost of available technologies even more striking. HgTe nanocrystals are among the most promising candidates to address SWIR since their spectrum can be tuned all over this range while demonstrating photoconductive properties. However, several main issues have been kept under the rug, which prevents further development of active materials and devices. Here we address two central questions, which are (i) the stability of the device under ambient air condition and (ii) the reduction of dark current. Encapsulation of HgTe CQDs is difficult because of their extreme sensitivity to annealing, we nevertheless demonstrate an efficient encapsulation method based on a combination of $\mathrm{O}_{2}$ and $\mathrm{H}_{2} \mathrm{O}$ repellant layers leading to stability over $>100$ days. Finally, we demonstrate that the dark current reduction can be obtained by switching from a photoconductive geometry to a photovoltaic (PV) device, which is fabricated using solution and low temperature based approach. We demonstrate fast photoresponse $(>10 \mathrm{kHz})$ and detectivity enhancement by 1 order of magnitude in the PV configuration at room temperature. These results pave the way for narrow bandgap CQD based cost-effective optoelectronic devices in developing next generation SWIR photonic systems.
\end{abstract}

To whom correspondence should be sent: el@insp.upmc.fr 


\section{INTRODUCTION}

Among potential applications of colloidal quantum dots (CQDs), their use for displays ${ }^{1}$ and solar cells ${ }^{2}$ have certainly generated the most of interest. ${ }^{3}$ For these two applications, relatively wide band gap nanocrystals (>1.2 eV) must be used. Nonetheless, CQDs are also quite promising for the emergence of low cost Infrared (IR) devices such as detectors and photon sources. IR electromagnetic radiations in short wave IR (SWIR; $1.4-3 \mu \mathrm{m}$ ) and in the two atmospheric transmission windows, mid wave IR (MWIR; $3-5 \mu \mathrm{m}$ ) and long wave IR (LWIR; $8-14 \mu \mathrm{m}$ ) are of utmost importance considering both space and civil applications; including earth environmental monitoring, night time surveillance, communications, etc. In particular, expansion to mass market of new applications, such as assistance to night driving or spectroscopic sorting, requires photodetectors with low price and relatively fast photoresponse. Current technologies are not able to achieve this combination, since quantum detectors are expensive and thermal detectors are slow. Lead chalcogenides ( $\mathrm{PbX}$ ) nanocrystals have been widely used to obtain absorption in the near infrared range. ${ }^{4,5}$ However, when longer wavelengths need to be addressed those materials are promptly limited by their bulk band gap. Even in the SWIR (0.8-3 $\mu \mathrm{m})$, the necessity of large PbX CQDs ${ }^{6}$ which are of poor colloidal stability, results in films with pinholes and the associated electrical shorts when going toward vertical geometry devices. In this sense, the use of confined semi-metal materials is of utmost interest to reach colloidally stable CQDs with narrower band gap. Mercury chalcogenides ( $\mathrm{HgX}$ ) have recently generated a significant interest for the design of IR devices ${ }^{7,8,9}$ in the range of wavelengths from near $\mathrm{IR}^{10}$, telecom ${ }^{11}(1.55 \mu \mathrm{m})$, mid IR ${ }^{12}$ and up to the $\mathrm{THz}^{13}$.

In this paper we focus on the so-called extended SWIR region where detection is conducted up to $2.5 \mu \mathrm{m}$ ( $4000 \mathrm{~cm}-1$ or $500 \mathrm{meV}$ ), which is of interest for night imaging based on night glow radiation. Even if the SWIR range is less demanding from a technological point of view than longer wavelength, its vicinity with the visible range makes the cost difference with visible technologies very striking. SWIR detection is currently driven by InGaAs technology, which suffers from its lack of spectral tunability and high cost. Thus, CQD based technologies may offer an interesting cost disruption for SWIR detection. ${ }^{14}$ However, some essential steps still have to be addressed before $\mathrm{HgX}$ nanocrystal based technologies can reach a mature level. In this paper, we address two of them, which are (i) the material and device stability toward air operation ${ }^{15,16}$ which is achieved using polymer encapsulation and (ii) dark current reduction, particularly keeping focus on room temperature operation, through the development of a photovoltaic device. For the reduction of dark current, we integrate narrow band gap HgTe CQDs with large band gap metal oxide $\left(\mathrm{TiO}_{2}\right)$ in stacked layers geometry.

\section{METHODS}

\section{Chemicals}

Mercury chloride $\left(\mathrm{HgCl}_{2}\right.$, Strem Chemicals, 99\%), tellurium powder (Te, Sigma-Aldrich, 99.99\%), trioctylphosphine (TOP, Cytek, 90\%), oleylamine (Acros, 80-90\%), dodecanethiol (DDT, Sigma-Aldrich, 98\%), 1,2,ethanedithiol (EDT, Fluka, 98.0\%), hydrochloric acid ( $\mathrm{HCl}$, Mieuxa, 25\%), lithium perchlorate $\left(\mathrm{LiClO}_{4}\right.$, Sigma-Aldrich, 98\%), polyethylene glycol (PEG, $\mathrm{M}_{\mathrm{w}}=6 \mathrm{~kg} \cdot \mathrm{mol}^{-1}$, Fluka), chloroform (Carlo Erba), ethanol absolute anhydrous (Carlo Erba, 99.9\%), methanol (Carlo Erba, 99.8\%), acetone (Carlo Erba, 99.8\%), n-hexane (Carlo Erba), n-octane (SDS, 99\%), toluene (Carlo Erba, 99.3\%), N, N-Dimethylformamide (DMF, > 99.8\%, Sigma Aldrich), Anatase titanium oxide nanoparticles $\left(\mathrm{TiO}_{2}\right.$, size: 8 $-10 \mathrm{~nm}$, Ti-Nanoxide HT-L/SC from Solaronix), Poly (vinyl alcohol) (PVA, Mw $=89000-98000 \mathrm{~kg} \cdot \mathrm{mol}^{-1}$, Sigma Aldrich), Poly (vinylidene fluoride) (PVDF, Mw $=180000 \mathrm{~kg} \cdot \mathrm{mol}^{-1}$, Sigma Aldrich), Poly(methyl methacrylate) (PMMA), Indium tin oxide (ITO) coated glass slide $\left(\rho_{\mathrm{s}}=70-100 \Omega / \mathrm{sq}\right.$, Sigma Aldrich). All chemicals are used as received, except oleylamine, which is centrifuged before used. Mercury compounds are highly toxic. Handle them with special care. 


\section{Material synthesis}

1 M TOP:Te precursor: $2.54 \mathrm{~g}$ of Te powder is mixed in $20 \mathrm{~mL}$ of TOP in a three neck flask. The flask is kept under vacuum at room temperature for $5 \mathrm{~min}$ and then the temperature is raised to $100{ }^{\circ} \mathrm{C}$. Furthermore, degassing of flask is conducted for the next $20 \mathrm{~min}$. The atmosphere is switched to $\mathrm{Ar}$ and the temperature is raised to $275^{\circ} \mathrm{C}$. The solution is stirred until a clear orange coloration is obtained. The flask is cooled down and the color switches to yellow. At room temperature, the flask is degassed again for $10 \mathrm{~min}$. Finally, this solution is transferred to an Ar filled glove box for storage.

HgTe CQD synthesis: In a $100 \mathrm{~mL}$ three-neck flask, $513 \mathrm{mg}$ of $\mathrm{HgCl}_{2}$ and $57.5 \mathrm{~mL}$ of oleylamine are degassed at $110^{\circ} \mathrm{C}$ for $1 \mathrm{~h}$. Under Ar at $80^{\circ} \mathrm{C}$, a solution containing $1.9 \mathrm{~mL}$ of $1 \mathrm{M}$ TOP:Te and $10 \mathrm{~mL}$ of oleylamine is quickly injected after warming it up with heat gun. After $3 \mathrm{~min}$, the reaction is quickly quenched by adding a mixture of toluene $(9 \mathrm{~mL})$ and DDT $(1 \mathrm{~mL})$, the flask is then cooled down using air flux. The nanocrystals are precipitated in ethanol and redispersed in toluene. This washing is repeated one more time. Finally, the HgTe CQDs dispersed in toluene are filtered through a $0.2 \mu \mathrm{m}$ PTFE filter. The obtained solution is used for further characterizations and fabrication of devices.

Electron microscopy: For TEM pictures, a drop of CQD solution is drop-casted on a copper grid covered with an amorphous carbon film. JEOL $2010 \mathrm{~F}$ is used at $200 \mathrm{kV}$ for acquisition of picture. For device characterization, FEI Magellan scanning electron microscope is used.

\section{Electrode fabrication}

Au/Glass electrode fabrication: Microscope glass slides $(75 \times 25 \times 1 \mathrm{~mm})$ are cut in half and cleaned by sonication in acetone and rinsed thoroughly with isopropanol, and then oxygen plasma cleaning is carried out for 5 minutes. An adhesion primer (TI-PRIME) is spin-coated onto each substrate before AZ5214E photoresist is spin-coated and baked at $110^{\circ} \mathrm{C}$ for $90 \mathrm{~s}$. A mask aligner is used to expose the substrates to UV light for $2 \mathrm{~s}$ through a lithography mask. Black paper is kept under the glass slides to prevent light from being reflected back to the photoresist. These substrates are baked at $125^{\circ} \mathrm{C}$ for $2 \mathrm{~min}$ to invert the photoresist and flood-exposed for $15 \mathrm{~s}$ with the mask aligner, again using black paper. Photoresist is developed using AZ726 developer for $20 \mathrm{~s}$ and rinsed with pure water. Patterned substrates are dried and cleaned with $5 \mathrm{~min}$ of oxygen plasma to remove photoresist residues. In a thermal evaporator, $5 \mathrm{~nm}$ of chromium is deposited as an adhesion promoter before $80 \mathrm{~nm}$ of gold is evaporated. Lift-off is conducted in an acetone bath for at least an hour. Electrodes are $2.5 \mathrm{~mm}$ long and spaced by 10 or $20 \mu \mathrm{m}$.

$\mathrm{Si} / \mathrm{SiO}_{2} / \mathrm{Au}$ electrodes: The surface of a $\mathrm{Si} / \mathrm{SiO}_{2}$ wafer ( $400 \mathrm{~nm}$ oxide layer) is cleaned by sonication in acetone. The wafer is rinsed with isopropanol and finally cleaned using an $\mathrm{O}_{2}$ plasma. AZ 5214E resist is spin-coated and baked at $110^{\circ} \mathrm{C}$ for $90 \mathrm{~s}$. The substrate is exposed under UV through a pattern mask for $2 \mathrm{~s}$. The film is further baked at $125^{\circ} \mathrm{C}$ for $2 \mathrm{~min}$ to invert the resist. Then a $40 \mathrm{~s}$ flood exposure is performed. The resist is developed using a bath of AZ 726 for $32 \mathrm{~s}$, before being rinsed in pure water. We then deposit a $5 \mathrm{~nm}$ chromium layer and $80 \mathrm{~nm}$ gold layer using a thermal evaporator. The lift-off is performed by dipping the film in acetone for $1 \mathrm{~h}$. The electrodes are finally rinsed using isopropanol and dried by an air flow. The electrodes are $2.5 \mathrm{~mm}$ long and spaced by $10 \mu \mathrm{m}$. These electrodes are used for photoconductive device and electrolyte gated transistor measurements.

Electrolyte gating: For electrolyte gating we first mix in a glove box, $0.5 \mathrm{~g}$ of $\mathrm{LiClO}_{4}$ with $2.3 \mathrm{~g}$ of PEG. The vial is heated at $170{ }^{\circ} \mathrm{C}$ on a hot plate for $2 \mathrm{~h}$ until the solution becomes clear. To use the electrolyte, the solution is warmed around $100{ }^{\circ} \mathrm{C}$ and brushed on the top of the HgTe CQD film.

\section{Photoconductive device preparation}


Ligand exchange: Inside a $\mathrm{N}_{2}$ filled glove-box, HgTe CQDs in toluene are drop-casted on pre-patterned interdigitated gold electrodes ( $10 \mu \mathrm{m}$ separation) on $\mathrm{SiO}_{2} / \mathrm{Si}$ substrates. After complete drying, EDT ligand exchange is performed by dipping the film in an EDT solution in ethanol ( $1-2 \mathrm{wt} \%)$ for $90 \mathrm{~s}$ and rinsing it in pure ethanol for $30 \mathrm{~s}$. This process is repeated for 3 to 4 times to get homogeneous and crack filled film with a device resistance of $100-200 \mathrm{k} \Omega$.

Protective layer of PMMA/PVA/PVDF: PMMA ( $5 \mathrm{wt} \%$ in $\mathrm{CHCl}_{3}$ ) solution is spin-coated on substrates at $2000 \mathrm{rpm}$ for $60 \mathrm{~s}$, then a quick annealing step at $50{ }^{\circ} \mathrm{C}$ for $1 \mathrm{~min}$ is performed. In following steps, PVA (centrifuged solution at 10 wt \% in water) and PVDF (10 wt \% in DMF) are spin coated at $4000 \mathrm{rpm}$ for $60 \mathrm{~s}$ and $1500 \mathrm{rpm}$ for $30 \mathrm{~s}$, respectively. After each step, substrates are annealed for $1 \mathrm{~min}$ at $50^{\circ} \mathrm{C}$. Finally, device is kept in vacuum overnight for the complete drying of encapsulation layers. Thus, obtained thicknesses for these encapsulation films are found to be $1.3 \mu \mathrm{m}, 0.5$ $\mu \mathrm{m}$ and $0.5 \mu \mathrm{m}$ of PMMA, PVA and PVDF, respectively.

\section{Photovoltaic device fabrication}

ITO patterning: ITO substrates are cut into $17 \times 17 \mathrm{~mm}$ size and thoroughly cleaned by sonication in acetone for $5 \mathrm{~min}$, rinsed with acetone and isopropanol, then dried completely with dry $\mathrm{N}_{2}$ flow. AZ 5214E photoresist is spin-coated for $30 \mathrm{~s}$ and subsequently the substrates are baked at $110{ }^{\circ} \mathrm{C}$ for $90 \mathrm{~s}$. At next stage, standard photolithography is performed using mask aligner for exposing the substrates to UV light for $5 \mathrm{~s}$ through a lithography mask ( $1 \mathrm{~mm}$ width). Photoresist is developed using AZ 726 developer for $40 \mathrm{~s}$ and immediately rinsed with de-ionized water. Thus, exposed ITO surface is completely etched out with $25 \% \mathrm{HCl}$ (in water) for 6 min and substrates are dipped immediately in deionized water. Then, lift-off is conducted in an acetone bath and patterned ITO substrates are cleaned with acetone and isopropanol. Finally, substrates are dried with dry $\mathrm{N}_{2}$ flow.

$\mathrm{TiO}_{2}$ film preparation: $200 \mu \mathrm{L}$ of anatase $\mathrm{TiO}_{2}$ nanoparticles solution is spin-coated on above patterned ITO substrates at $5000 \mathrm{rpm}$ for $30 \mathrm{~s}$. The $\mathrm{TiO}_{2}$ film is annealed at $200{ }^{\circ} \mathrm{C}$ for $15 \mathrm{~min}$ and its thickness is measured to be $65 \mathrm{~nm}$ with Dektak profilometer.

HgTe CQD film preparation and EDT ligand exchange in air free conditions: Film preparation and ligand exchange are carried out inside a $\mathrm{N}_{2}$ filled glovebox. In a typical procedure, $80 \mu \mathrm{L}$ of concentrated HgTe CQDs $(25 \mathrm{mg} / \mathrm{mL})$ from toluene is spin coated at $2000 \mathrm{rpm}$ for $30 \mathrm{~s}$ on above fabricated $\mathrm{Glass} / \mathrm{ITO} / \mathrm{TiO}_{2}$ substrates. After complete evaporation of solvent, ligand exchange is carried out by dipping the film in $1-2 \mathrm{wt} \%$ EDT solution in ethanol for $90 \mathrm{~s}$ and rinsing it in pure ethanol for $30 \mathrm{~s}$. Afterwards, a quick annealing step at $50{ }^{\circ} \mathrm{C}$, for $1 \mathrm{~min}$ is carried out. This procedure is repeated for $8-9$ times to get thicker $(180-200 \mathrm{~nm})$ and pin-hole free HgTe CQD film.

Au electrode deposition in air free conditions: Fabricated Glass/ITO/ $\mathrm{TiO}_{2} / \mathrm{HgTe}$ substrates were transferred from glove box to the thermal evaporator chamber under $\mathrm{N}_{2}$ environment. $80 \mathrm{~nm}$ thick Au was evaporated at a rate of $2-$ $5 \mathrm{~A} / \mathrm{s}$ using shadow mask ( $1 \mathrm{~mm}$ width) technique and the mask was aligned to get a pixel of $1 \times 1 \mathrm{~mm}$ area.

Encapsulation of device: Finally, the fabricated device is transferred back to the glove box under $\mathrm{N}_{2}$ environment. The PMMA/PVA/PVDF encapsulation films are deposited on top of device with the above mentioned protocol and the device is kept in vacuum overnight for the complete drying of encapsulation layers.

\section{Electrical measurements}

DC transport: The sample is connected to a Keithley 2634b, which controls the drain bias ( $\left.\mathrm{V}_{\mathrm{DS}}\right)$ between -2 and $+2 \mathrm{~V}$ with a step of $10 \mathrm{mV}$, and measures the associated current (IDs). This measurement is made in the dark and under illumination using diodes ( $405 \mathrm{~nm}$, power $=0.17-22 \mathrm{~mW}, 532 \mathrm{~nm}$, power $=25 \mathrm{~mW}$ (used for inside the glove box) and $635 \mathrm{~nm}$, power $=7 \mathrm{~mW}$ (used for in air measurement). 
Transistor measurements: The sample is connected to a Keithley $2634 \mathrm{~b}$, which sets the drain-source bias $\left(V_{D S}=10\right.$ $\mathrm{mV})$, controls the gate bias ( $\left.\mathrm{V}_{\mathrm{GS}}\right)$ between -2 and $+2 \mathrm{~V}$ with a step of $1 \mathrm{mV}$ and measures the associated currents $\mathrm{I}_{\mathrm{DS}}$ and $I_{G s}$. The measurements are repeated two times to ensure that the obtained curves are stable.

Setup and experimental condition for transient photocurrent, noise measurements and frequency dependence of the photocurrent are given in Figure S2, S7 and S8, respectively.

\section{RESULTS and DISCUSSION}

To start our discussion, we focus on the active material. We synthetize HgTe CQDs with a band edge energy at 4000 $\mathrm{cm}^{-1}$ using the Keuleyan's procedure ${ }^{17}$, see experimental section and Figure 1C. CQDs are actually tetrapods, see transmission electron microscopy (TEM) in Figure 1a-b. Initially, the HgTe CQDs are capped with long thiol chains (dodecanethiol: DDT), which ensures the colloidal stability of the particle. To build photoconductive film of HgTe, the exchange of these long native ligands toward shorter molecules is very critical. Herein, we used solid state ligand exchange for ethanedithiol (EDT) molecules, since we demonstrate recently that short dithiols are the best tradeoff between enhancing the mobility by carrier lifetime product and the amount of introduced traps. ${ }^{18}$ After ligand exchange, we observe a broadening of the excitonic feature, as well as a significant reduction of the peak relative to the $\mathrm{C}-\mathrm{H}$ bond $\left(\approx 3000 \mathrm{~cm}^{-1}\right)$, which confirms the efficiency of the DDT removal, see Figure 1c.
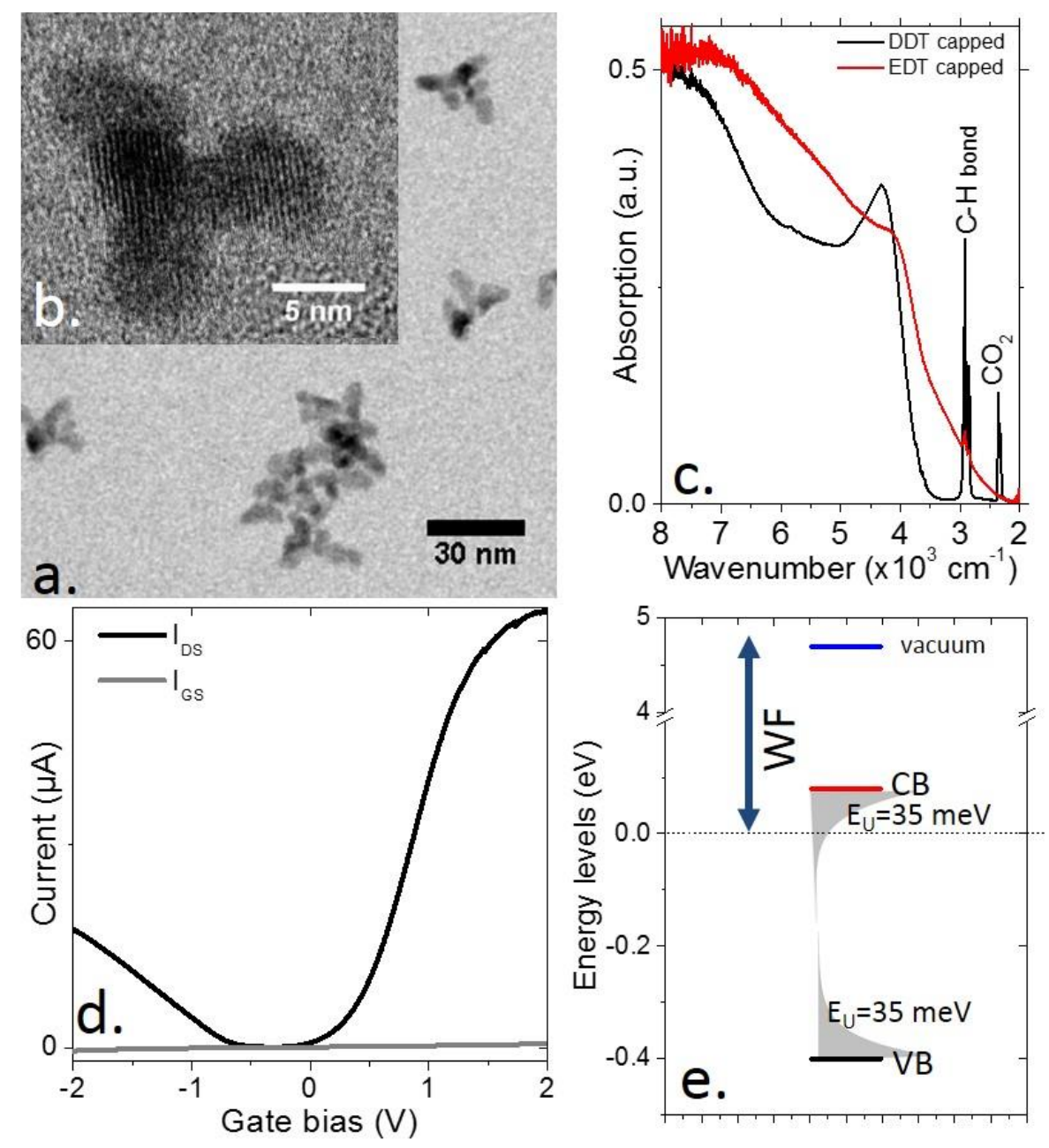

Figure 1 - $a$. and b. are low and high magnification TEM images of HgTe nanocrsytals, respectively. c. Absorption spectrum of DDT-capped and EDT-capped HgTe CQDs. $d$. Transfer curve (drain current as a function of gate bias) for an HgTe CQD film capped with EDT ligand probed in an electrolyte gated transistor configuration. e. Reconstructed 
energy spectrum of HgTe CQDs from XPS data (see Supplementary Informations). The Fermi level corresponds to $0 \mathrm{eV}$ energy level. Adapted with permission from ref 18. Copyright (2018) American Chemical Society.

Transport properties probed in an electrolyte gated field effect transistor configuration ${ }^{19}$ reveals an ambipolar behavior showing the ability of HgTe CQD film for both hole and electron conduction ${ }^{20}$, see Figure $1 \mathrm{~d}$. The hole mobility is typically three times lower than the electron mobility.

To finish those preliminary results about the material, we have measured its electronic spectrum in absolute energy scale, see Figure 1e. The band edge energy is measured though the IR spectrum, see Figure 1c. The positions of the Fermi level and valence band from the vacuum level are determined from photoemission measurements conducted on the TEMPO beamline of synchrotron Soleil. The Fermi level lies in the upper part of the band gap ( $80 \mathrm{meV}$ below the conduction band), which confirms the slightly more n-type nature of the material observed in transport measurement, while the vacuum level is typically $4.7 \mathrm{eV}$ above the Fermi level, see Figure S1. From transient photocurrent measurement, see Figure $\mathrm{S} 2$, we are able to determine the Urbach energy (i.e. the characteristic energy decay of the trap distribution within the band gap) to be $35 \mathrm{meV} .{ }^{18}$ This relatively low value is critical to obtain reasonable open circuit voltage, ${ }^{21,22}$ with in mind the integration of the HgTe CQDs into a photovoltaic configuration device.

While the film presents conductive and photoconductive behaviors even in air ${ }^{15,18,23}$ its properties are far from stable. Even if the film is prepared in air free condition (i.e. in glove box) the conductance rises once exposed to air, see Figure 2a. This increase of the dark conductance just after exposure is not even leading to a plateau and we keep observing an increase over the next 10 days, see Figure 2e, indicating the severe degradation of the device overtime. This increase in dark conductance will be quite detrimental for photodetector device fabrication. An usual solution to overcome a large dark current is to cool down the sample to reduce the amount of thermally activated carriers. However, ligand exchanged HgTe CQD film reveals a non-monotonic behavior of the I-T curve, ${ }^{15}$ see Figure $2 \mathrm{~b}$, where cooling the sample can even increase the dark conductance. It was previously demonstrated ${ }^{15}$ that air free preparation and handling of the film prevents such degradation of the performance. To address this issue we developed an alternative solution by encapsulating the film using protective layers. Encapsulation of CQDs has recently become a major topic in particular for their use in displays. In this case, the goal of the encapsulation is to preserve the photoluminescence efficiency at high temperature, while preventing oxygen and water exposure. This can be achieved by using polymer ${ }^{1}$ or inorganic matrix ${ }^{24}$ grown in solution or by physical method such as by ALD $^{25,26}$ or PLD $^{27}$.

However, in the case of HgTe CQDs, most of these methods cannot be used. The previously mentioned methods require mild temperature $\left(100-200^{\circ} \mathrm{C}\right)$ annealing operations, which leads to irreversible changes in the $\mathrm{HgTe}$ CQD film. Figure S3a shows that above $50{ }^{\circ} \mathrm{C}$, annealing of the $\mathrm{HgTe}$ CQD film leads to a significant broadening of the exciton absorption. HgTe has a much larger Bohr radius ( $40 \mathrm{~nm}$ ) than many other CQD material ( $\approx 7 \mathrm{~nm}$ for CdSe or $\left.\mathrm{CsPbBr}_{3}\right)$, and as a result HgTe CQDs are strongly confined and thus very sensitive to annealing, which leads to a partial sintering of the nanoparticles. The consecutive change of particle size makes the electrical band gap of the material becoming driven by the tail absorption. As a result, thermal activation of carriers becomes even more dramatic and we observe $a$ rise of the conductance, see Figure $2 a-b$ and S4. Thus, only quasi room temperature conditions can be used in the case of $\mathrm{HgTe}$ CQDs. We choose a strategy to combine $\mathrm{O}_{2}$ and $\mathrm{H}_{2} \mathrm{O}$ insulating barrier polymer layers to prevent any oxidation of the HgTe CQD film. All steps of film preparation (deposition + ligand exchange) are conducted in air free condition. Still in glove box, a thick $(1.3 \mu \mathrm{m})$ PMMA layer is spin coated to obtain a smooth surface. Then a PVA layer $(500 \mathrm{~nm})$ is used as oxygen repealing material, before a PVDF layer $(500 \mathrm{~nm})$ gets deposited as water insulator, see a scheme of the device as inset of Figure $2 c$. With such encapsulation system, no rise of the conductance is observed while the system is brought to air environment, see Figure $2 \mathrm{c}$ and e. Moreover, the temperature dependence of the film recovers a monotonic behavior, see Figure $2 \mathrm{~d}$. The Arrhenius fit of the I-T curve around room temperature leads to an activation energy of $114 \mathrm{meV}$. With such encapsulation, stable dark current and photoresponse are observed over several month time scale, see Figure 2e-f. 

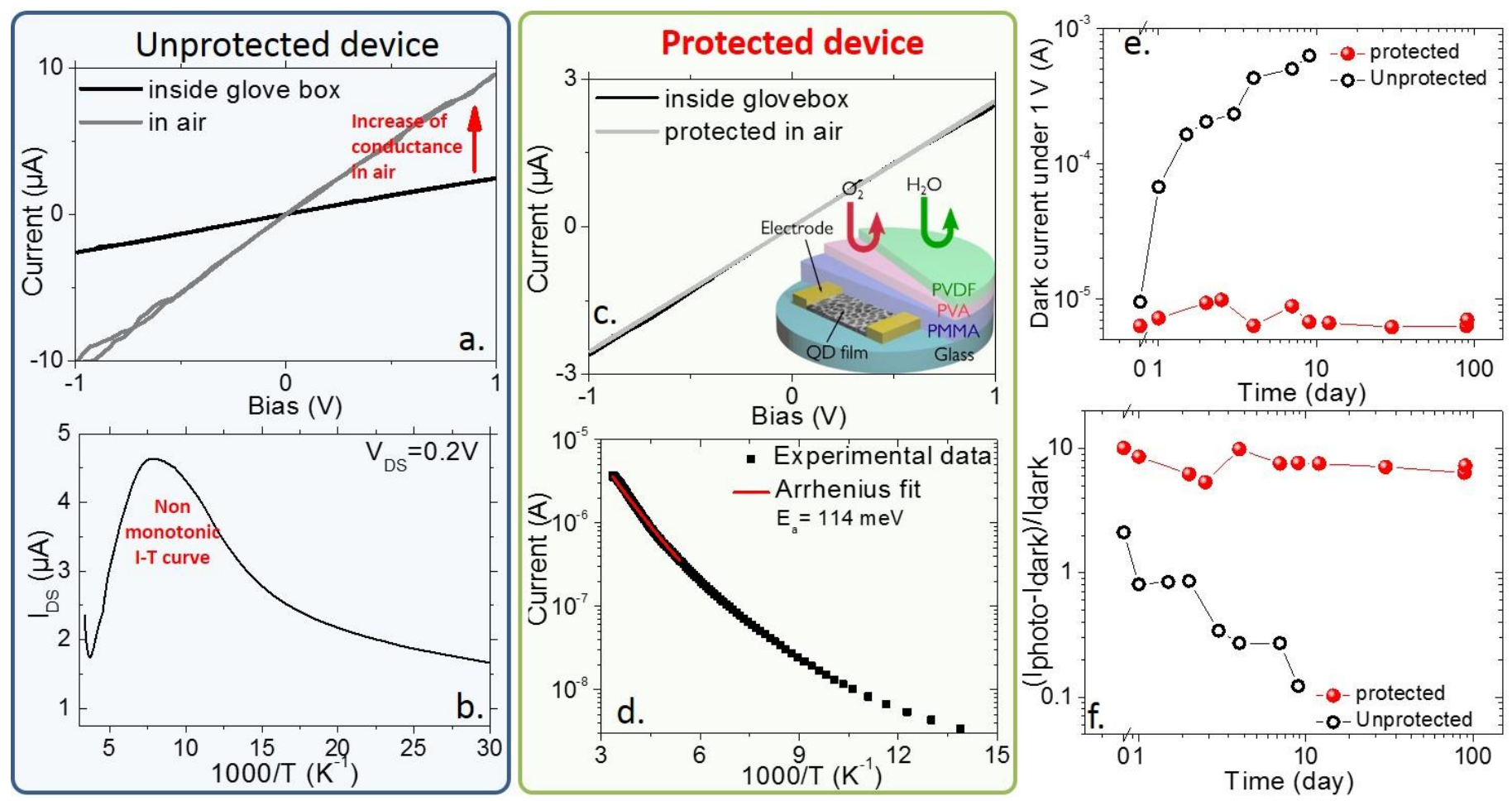

Figure 2 - $a . I-V$ curves of a HgTe CQD film prepared and measured in glove box and after (next hour) air exposure. $b$. I-T curve of an HgTe CQD film after the film has been exposed to air. C. I-V curves of a HgTe CQD film, prepared with protective layers, and measured in glove box and after air exposure. Inset is a scheme of the HgTe CQD film device with protective layers. d. I-T curve of an HgTe CQD film prepared with protective layers, after the film has been exposed to air. e. Dark current as a function of time spent in air for a HgTe CQD film with and without protective layers. Time zero corresponds to the sample in the air-free glove box. $f$. Ratio of photocurrent over dark current as a function of time spent in air for HgTe CQD films with and without protective layers.

The full characterization of the protected device in planar configuration is given in figure $\mathrm{S} 9$ and Figure 3 . The device presents a responsivity ( $R$ in $A . W^{-1}$ ) in this configuration reaching $10 \mathrm{~mA} . W^{-1}$ (Figure 3a), which corresponds to internal quantum efficiency of $3 \%$. The device present a fast response, with a $3 \mathrm{~dB}$ bandwidth above $10 \mathrm{kHz}$ (Figure $3 \mathrm{~b}$ ), which is compatible requested dynamics for imaging. The noise current density $\left(i_{n}\right.$ in $\left.A . H z^{-1 / 2}\right)$ is limited by $1 / f$ noise as expected for HgTe CQD film ${ }^{23,28,29}$, see figure S9c, and is typically in the range of $\mathrm{nA} \cdot \mathrm{Hz}^{-1 / 2}$. The specific detectivity $\mathrm{D}^{*}$ (i.e. signal to noise ratio) defined as $D^{*}=\frac{R \sqrt{A}}{i^{n}}$ with $A$ the optical area is typically at room temperature around a few $10^{6}$ jones, see Figure $3 d$. 

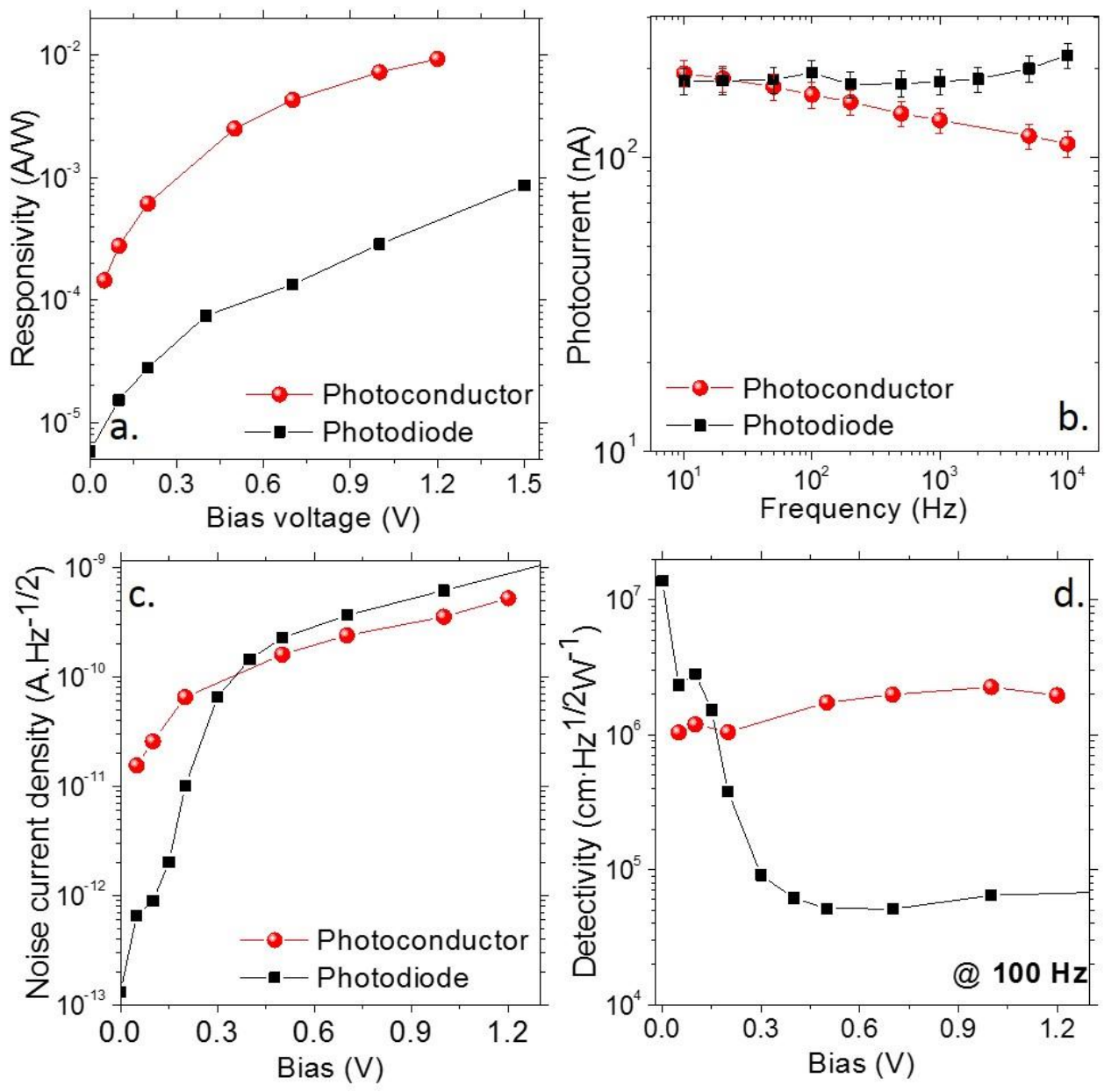

Figure 3 -Performances of protected photodetectors in photoconductive and photodidoe configuration a. Responsivity as a function of the applied bias under blackbody illumination $\left(T_{B B}=927^{\circ} \mathrm{C}\right.$ ) for a HgTe CQD film. b. Bode diagram of the photocurrent intensity for a HgTe CQD film, as a function of the light chopping frequency. c. Noise current density (at $100 \mathrm{~Hz}$ ) as a function of applied bias for a HgTe CQD film. d. Room temperature detectivity at $100 \mathrm{~Hz}$, as a function of the applied bias for a HgTe CQD film.

One way to increase performance is to cool down the device. Assuming a weak temperature dependence of the mobility and given the I-T curve of Figure $2 \mathrm{~d}$, we estimate that detectivity around $10^{8}$ jones can be obtained for an operating temperature of $125 \mathrm{~K}$. However, to push even further the performances, it is critical to design device geometry with inherently less dark current. Switching from photoconductive mode to photovoltaic (PV) mode is a proven way to reduce the applied bias over the sample and thus the dark current. ${ }^{30}$ Indeed the built in bias of the photodiode allows to operate the device at zero (or very reduced) bias, where the dark current and its associated noise should be reduced. While PbS CQDs have been intensively integrated to PV geometry, far less work have been focused on $\mathrm{HgTe}^{31,32,33}$ and especially in the SWIR range. ${ }^{34}$ Actually glass/ITO extensively used for solar cell remains quite transparent in the SWIR range, as long as thin ITO layer are used, see figure S5. Thus, we choose to use this substrate with in mind the design of a low-cost device. Because of the pretty large work function (4.7 eV), ITO can be used as electron injector and an inverted diode structure can be built. Our PV device is made of a stack of ITO/TiO $/ \mathrm{HgTe} / \mathrm{Au}$. Since the $\mathrm{TiO}_{2}$ layer typically requires an annealing step, this configuration presents the benefit to limit the exposure of HgTe QD to high temperature. The HgTe layer typically presents a $180-200 \mathrm{~nm}$ thickness and is obtained through a multilayer deposition process to ensure an efficient ligand exchange and also to fill up any possibly formed cracks, see image of the device in Figure 4a. Finally a gold top contact is used as hole extractor with a high stability toward oxygen and water exposure. 
The cross sectional scanning electron microscopy (SEM) image (Figure 4a) confirms the high quality stacking of $\mathrm{TiO}_{2} / \mathrm{HgTe}$ CQD layers on ITO substrates. To obtain an air stability operation, the device is further encapsulated using the earlier proven PMMA/PVA/PVDF stack of polymer layers. A picture of the final device is shown as inset of Figure 4a. The encapsulating layers are gently removed from the external part of the gold contact (ie away from the active device) using a razor blade and cotton swab to be able to connect electrical contact.

To understand the charge transport in this device, its I-V characteristics was measured, see Figure $4 \mathrm{~b}$, and it clearly shows a rectifying behavior, at room temperature. Photocurrent can be generated even at zero bias, see Figure $4 \mathrm{~b}$.

Figure 4c compares the current modulation induced by the light for photoconductive and PV devices in two configurations. In the photoconductive mode, we see a limited current increase under illumination, see Figure 4c. The signal to noise ratio is actually limited by the large background signal (i.e. the dark current). As PV mode is used, we observe a huge decrease of the background, thanks to zero bias operation; see Figure 4b.

The key advantage of the PV geometry compared to photoconductive operation is the increase of photocurrent to dark current ratio, as shown in Figure 4c. For the same light power density, we notice an increase of the ratio by a factor $>70$, as going toward the PV geometry.
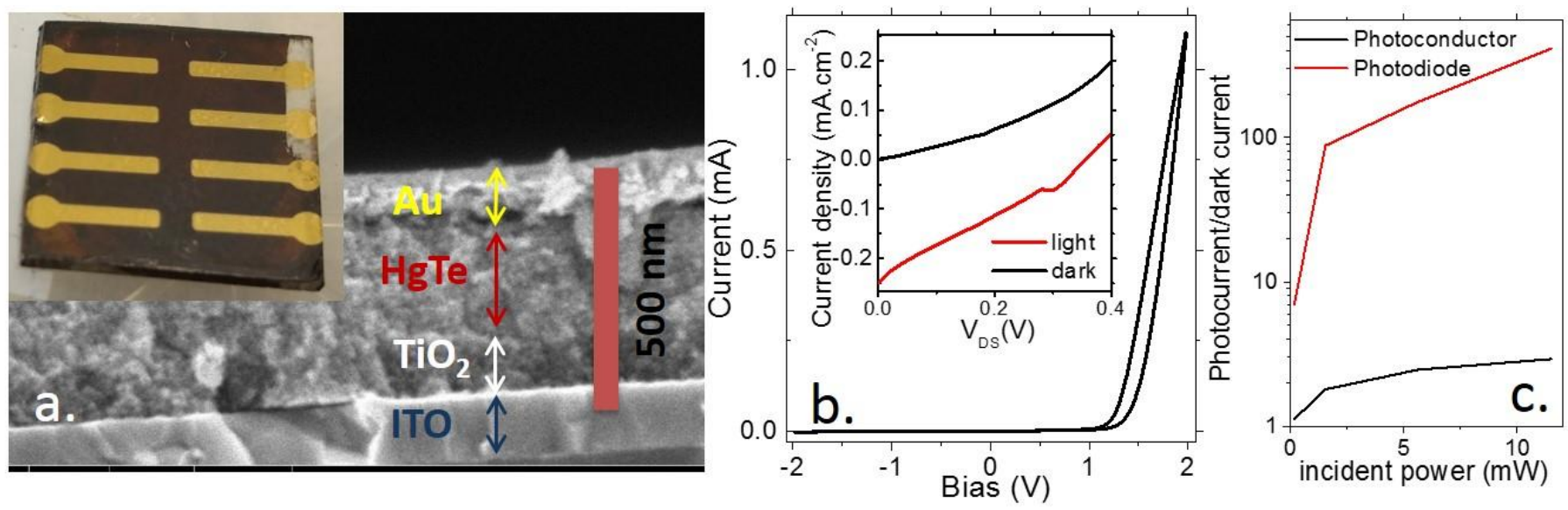

Figure 4-a. Cross sectional SEM image of a HgTe CQD device in photovoltaic configuration. Inset is a top view of the final device. $b . I-V$ curve of the HgTe CQD device in photovoltaic configuration. Inset is a zoom in the zero bias region under dark condition and under illumination by a blue $(405 \mathrm{~nm})$ laser with a $22 \mathrm{~mW}$ power. C Photocurrent over dark current ratio as a function of incident power for the photoconductive device (Au/HgTe/Au) and for the PV device with an $\mathrm{ITO} / \mathrm{TiO}_{2} / \mathrm{HgTe} / \mathrm{Au}$ geometry.

Performances of this PV device are reported in Figure 3 and S10 for full characterization. We would like to stress the main difference with the photoconductive geometry. First we observe a drops of the photoreponse by one order of magnitude, see Figure 3a. The latter is not due to absorption of electrode in the SWIR range, but actually due to the limited internal quantum efficiency of our device, typically $0.1 \%$, as shown in figure $\mathrm{S} 11$. This demonstrates that charge extraction is still not optimized. On the other hand, the promptness of the photoresponse is preserved with a $3 \mathrm{~dB}$ bandwidth still above the capacity of the current setup $(10 \mathrm{kHz})$, see Figure $3 \mathrm{~b}$. As it was expected for PV geometry we notice a drastic decrease of the noise current density at low bias by more than two decades with respect to the photoconductive geometry, see Figure 3c. This results in an enhanced detectivity, which now reach $10^{7}$ jones at room temperature. Finally the stability of the device has been checked, and the I-T curve (see figure S4c) is similar to the one obtained for photoconductive devices with protective layer, while the photodetection performance remains unchanged at least over 7 weeks.

\section{CONCLUSIONS}


We report a solution based procedure, allowing air stable room temperature operation of HgTe nanocrystals for photodetection in SWIR range over several month time scale. The procedure is based on a low temperature $\left(50^{\circ} \mathrm{C}\right)$ approach to prevent any sintering of nanocrystals, which may potentially reduce the equivalent band gap and rise the device dark current. We demonstrate that a combination of PMMA/PVA/PVDF polymer layers prevents the previously observed degradation of performances (i.e. rise of conductance in air and non-monotonic I-T curve). We also demonstrate that PV device geometry can be effectively used to reduce the device dark current at room temperature and obtain an increase of the photocurrent to dark current ratio by a factor of 70 . In all geometries, HgTe leads to fast photoresponse, with associated bandwidth above $10 \mathrm{kHz}$, which is enough for most imaging applications. By switching from photoconductive to photovoltaic device geometry, detectivity in SWIR region is found to be enhanced by 1 order of magnitude at room temperature. These results will pave the way for long term device stability and solution-based approach for promising next generation SWIR optoelectronics based on narrow bandgap HgTe CQDs.

\section{SUPPORTING INFORMATIONS}

Supporting information include details about Photoemission measurements, experimental setup for device characterization (transient photocurrent, noise, frequency response), material characterizations (temperature dependence), device and structure characterizations (optical properties of the device, band alignment)

\section{ACKNOWLEDEGMENTS}

EL thanks the support of ERC starting grant blackQD. We acknowledge the use of clean-room facilities from the "Centrale de Proximité Paris-Centre". This work has been supported by the Region lle-de-France in the framework of DIM Nano-K (grant dopQD). This work was supported by French state funds managed by the ANR within the Investissements d'Avenir programme under reference ANR-11-IDEX-0004-02, and more specifically within the framework of the Cluster of Excellence MATISSE and also by the grant Nanodose and H2DH. NG and AJ thank Nexdot for post doc funding.

\section{REFERENCES}

\footnotetext{
${ }^{1}$ Xie., B.; Hun, R.; Xiaobing, L. Quantum Dots-Converted Light-Emitting Diodes Packaging for Lighting and Display: Status and Perspectives. J. Electron. Package. 2016, $138,020803$.

2 Konstantatos, G.; Sargent, E. H. Colloidal Quantum Dot Optoelectronics and Photovoltaics; Cambridge University Press, 2013.

${ }^{3}$ Talapin, D. V.; Lee, J. S.; Kovalenko, M. V.; Shevchenko, E. V. Prospects of Colloidal Nanocrystals for Electronic and Optoelectronic Applications. Chem. Rev. 2010, 110, 389-458.

${ }^{4}$ Hines, M. A.; Scholes, G.D. Colloidal PbS Nanocrystals with Size-Tunable Near-Infrared Emission: Observation of PostSynthesis Self-Narrowing of the Particle Size Distribution. Adv. Mater. 2013, 15, 1844-1849.

${ }^{5}$ McDonald, S. A.; Konstantatos, G.; Zhang, S.; Cyr, P. W.; Klem, E. J. D.; Levina, L. Sargent, E. H. Solution-Processed PbS Quantum Dot Infrared Photodetectors and Photovoltaics. Nat. Mater. 2005, 4, 138-142.

${ }^{6}$ Pietryga, J. M.; Schaller, R. D.; Werder, D.; Stewart, M. H.; Klimov, V. I.; Hollingsworth, J. A. Pushing the Band Gap Envelope: Mid-Infrared Emitting Colloidal PbSe Quantum Dots. J. Am. Chem. Soc. 2004, 126, 11752-11753.

${ }^{7}$ Kershaw, S. V.; Susha, A. S.; Rogach, A. L. Narrow Bandgap Colloidal Metal Chalcogenide Quantum Dots: Synthetic Methods, Heterostructures, Assemblies, Electronic and Infrared Optical Properties. Chem. Soc. Rev. 2013, 42, 3033 3087.

${ }^{8}$ Lhuillier, E.; Guyot Sionnest, P. Recent Progresses in Mid Infrared Nanocrystal Based Optoelectronics. IEEE J. Sel. Top. Quantum Electron. 2017, 23, 1-8.
} 
9 Keuleyan, S.; Lhuillier, E.; Brajuskovic, V.; Guyot-Sionnest, P. Mid-Infrared HgTe Colloidal Quantum Dot Photodetectors. Nat. Photonics 2011, 5, 489-493.

${ }^{10}$ Livache, C.; Izquierdo, E.; Martinez, B.; Dufour, M.; Keuleyan, S.; Pierruci, D.; Cruguel, H.; Becerra, L.; Fave, J. L; Aubin, H.; Ouerghi, A.; Lacaze, E.; Silly, M.; Dubertret, B.; Ithurria, S.; Lhuillier, E. Charge Dynamics and Optolectronic Properties in HgTe Colloidal Quantum Wells. Nano Lett. 2017, 17, 4067-4074.

${ }^{11}$ Kovalenko, M. V.; Kaufmann, E.; Pachinger, D.; Roither, J.; Huber, M.; Stangl, J.; Hesser, G.; Schäffler, F.; Heiss, W. Colloidal HgTe Nanocrystals with Widely Tunable Narrow Band Gap Energies: From Telecommunications to Molecular Vibrations. J. Am. Chem. Soc. 2006, 128, 3516-3517.

12 Tang, X.; Tang, X.; Lai, K. W. C. Scalable Fabrication of Infrared Detectors with Multispectral Photoresponse Based on Patterned Colloidal Quantum Dot Films. ACS Photonics 2016, 3, 2396-2404.

${ }^{13}$ Lhuillier, E.; Scarafagio, M.; Hease, P.; Nadal, B.; Aubin, H.; Xu, X. Z.; Lequeux, N.; Patriarche, G.; Ithurria, S.; Dubertret, B. Infrared Photodetection Based on Colloidal Quantum-Dot Films with High Mobility and Optical Absorption up to THz. Nano Lett. 2016, 16, 1282-1286.

${ }^{14}$ Chen, M.; Lu, H.; Abdelazim, N. M.; Zhu, Y.; Wang, Z.; Ren, W.; Kershaw, S. V.; Rogach, A. L.; Zhao, N. Mercury Telluride Quantum Dot Based Phototransistor Enabling High-Sensitivity Room-Temperature Photodetection at 2000 nm. ACS Nano 2017, 11, 5614-5622.

${ }^{15}$ Lhuillier, E.; Keuleyan, S.; Zolotavin, P.; Guyot-Sionnest, P. Mid-Infrared HgTe/As $\mathrm{S}_{3}$ FETs and Photodetectors. Adv. Mat. 2013, 25, 137-141.

${ }^{16}$ Chen, M.; Yu, H.; Kershaw, S. V.; Xu, H.; Gupta, S.; Hetsch, F.; Rogach, A. L.; Zhao, N. Fast, Air-Stable Infrared Photodetectors based on Spray-Deposited Aqueous HgTe Quantum Dots. Adv. Funct. Mater. 2014, 24, 53-59.

${ }^{17}$ Keuleyan, S.; Lhuillier, E.; Guyot-Sionnest, P. Synthesis of Colloidal HgTe Quantum Dots for Narrow Mid-IR Emission and Detection. J. Am. Chem. Soc. 2011, 133, 16422-16424.

${ }^{18}$ Martinez, B.; Livache, C.; Goubet, N.; Jagtap, A.; Cruguel, H.; Lacaze, E.; Silly, M. G.; Lhuillier, E. Probing Charge Carrier Dynamics to Unveil the Role of Surface Ligands on HgTe Narrow Band Gap Nanocrystals, J. Phys. Chem. C 2018, 122, 859-865.

${ }^{19}$ Lhuillier, E.; Ithurria, S.; Descamps-Mandine, A.; Douillard, T.; Castaing, R.; Xu, X.Z.; Taberna, P. L.; Simon, P.; Aubin, H.; Dubertret, B. Investigating the n- and p-Type Electrolytic Charging of Colloidal Nanoplatelets. J. Phys. Chem. C 2015, 119, 21795-21799.

${ }^{20}$ Liu, H.; Keuleyan, S.; Guyot-Sionnest, P. n-and p-Type HgTe Quantum Dot Films. J. Phys. Chem. C 2011, 116, 13441349.

${ }^{21}$ Chuang, C. H. M.; Maurano, A.; Brandt, R. E.; Hwang, G. W.; Jean, J.; Buonassisi, T.; Bulović, V.; Bawendi; M. G. OpenCircuit Voltage Deficit, Radiative Sub-Bandgap States, and Prospects in Quantum Dot Solar Cells. Nano Lett. 2015, 15, 3286-3294.

${ }^{22}$ Zhitomirsky, D.; Voznyy, O.; Levina, L.; Hoogland, S.; Kemp, K. W.; Ip, A. H.; Thon, S. M.; Sargent, E. H. Engineering Colloidal uantum Dot Solids Within and Beyond the Mobility-Invariant Regime. Nat. Commun. 2014, 5, 3803-3810.

${ }^{23}$ Lhuillier, E.; Keuleyan, S.; Rekemeyer, P.; Guyot-Sionnest, P. Thermal Properties of Mid Infrared Colloidal Quantum Dot Detectors. J. Appl. Phys. 2011, 110, 033110-033118.

${ }^{24}$ Wang, C. F.; Fan, F.; Sabatini, R. P.; Voznyy, O.; Bicanic, K.; Li, X.; Sellan, D. P.; Saravanapavanantham, M.; Hossain, N.; Chen, K.; Hoogland, S.; Sargent, E. H. Quantum Dot Color-Converting Solids Operating Efficiently in the $\mathrm{kW} / \mathrm{cm}^{2}$ Regime. Chem. Mater. 2017, 29, 5104-5112.

${ }^{25}$ Loiudice, A.; Saris, S.; Oveisi, E.; Alexander, D. T. L.; Buonsanti, R. CsPbBr 33 QD/AlOx Inorganic Nanocomposites with Exceptional Stability in Water, Light, and Heat. Angew. Chem. Int. Ed. Engl. 2017, 56, 10696-10701.

${ }^{26}$ Pourret, A.; Guyot-Sionnest, P.; Elam, J. W. Atomic Layer Deposition of ZnO in Quantum Dot Thin Films. Adv. Mater. 2009, 21, 232-235.

${ }^{27}$ Aubret, A.; Houel, J.; Pereira, A.; Baronnier, J.; Lhuillier, E.; Dubertret, B.; Dujardin, C.; Kulzer, F.; Pillonnet, A. Nondestructive Encapsulation of CdSe/CdS Quantum Dots in an Inorganic Matrix by Pulsed Laser Deposition. ACS Appl. Mater. Interfaces 2016, 8, 22361-22368.

${ }^{28}$ Liu, H.; Lhuillier, E.; Guyot-Sionnest, P. 1/f Noise in Semiconductor and Metal Nanocrystal Solids. J. Appl. Phys. 2014, $115,154309-154316$.

${ }^{29}$ De lacovo, A.; Venettacci, C.; Colace, L.; Scopa, L.; Foglia, S. Noise Performance of PbS Colloidal Quantum Dot Photodetectors. Appl. Phys. Lett. 2017, 111, 211104 - 211109.

${ }^{30}$ Guyot-Sionnest, P.; Roberts, J. A. Background Limited Mid-Infrared Photodetection with Photovoltaic HgTe Colloidal Quantum Dots. Appl. Phys. Lett. 2015, 107, $253104-253109$.

${ }^{31} \mathrm{Im}$, S. H.; Kim, H. J.; Kim, S. W.; Kim, S.W.; Seok, I. I. Efficient HgTe Colloidal Quantum Dot-Sensitized Near-Infrared Photovoltaic Cells. Nanoscale 2012, 4, 1581-1584. 
32 O'Connor, É.; O’Riordan, A.; Doyle, H.; Moynihan, S.; Cuddihy, A.; Redmond, G. Near-Infrared Electroluminescent Devices Based on Colloidal HgTe Quantum Dot Arrays. Appl. Phys. Lett. 2005, 86, 201114-201116.

${ }^{33}$ Günes, S.; Neugebauer, H.; Sariciftci, N. S.; Roither, J.; Kovalenko, M. V.; Pillwein, G.; Heiss, W. Hybrid Solar Cells Using HgTe Nanocrystals and Nanoporous $\mathrm{TiO}_{2}$ Electrodes, Adv. Funct. Mater. 2006, 16, 1095-1099.

${ }^{34}$ Chen, M.; Shao, L.; Kershaw, S. V.; Yu, H.; Wang, J.; Rogach, A. L.; Zhao, N. Photocurrent Enhancement of HgTe Quantum Dot Photodiodes by Plasmonic Gold Nanorod Structures. ACS Nano 2014, 8, 8208-8216. 
TOC Graphic

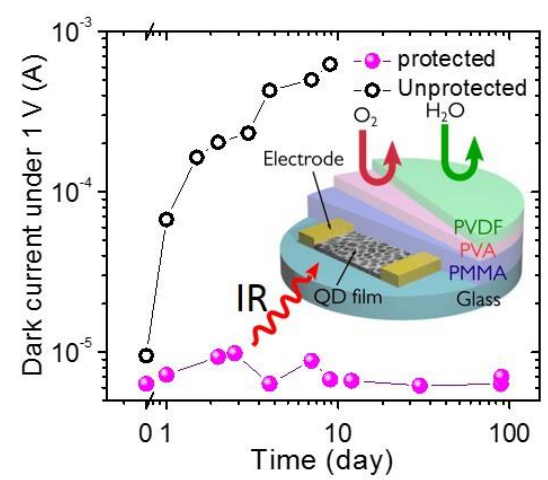

